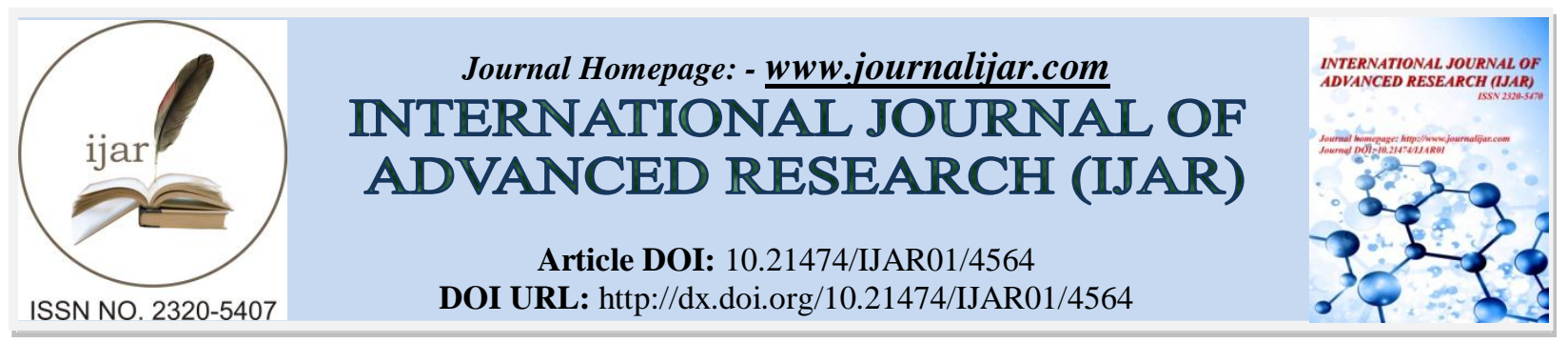

RESEARCH ARTICLE

\title{
CHARACTER RECOGNITION BASED ON SKELETONIZATION: A SURVEY.
}

\author{
Chetan A. Sultane, Milind V. Bhalerao, Sanjiv V. Bonde. \\ Department of E\&TC, SGGSIE \& T, Nanded.
}

\section{Manuscript Info}

Manuscript History

Received: 22 April 2017

Final Accepted: 24 May 2017

Published: June 2017

Key words:-

Pattern recognition, character recognition, skeletonization algorithm, Preprocessing, Segmentation, Feature extraction, classification, execution time

\section{Abstract}

In machine learning and pattern recognition the development of character recognition is an interesting area since 1970. There have been significant improvements in the research related to the recognition of printed as well as handwritten characters. Skeletonization is a crucial step in many digital image processing applications like palm recognition, medical imaging, fingerprint classification, etc. In the character recognition generally steps to be followed are preprocessing, segmentation, feature extraction, classification, and post-processing. There have been a number of methods and algorithm based on the skeletonization techniques is present in every step of character recognition process which is used to measure the parameters such as a number of connected components, skeletonization, thinning rate, and execution time, performance evaluation, etc. In this paper, different skeletonization algorithm for print as well as handwritten text in each step of character recognition is summarized and surveyed which algorithm is the best choice for the particular application and in future how this will be useful for the Devanagari character and digits recognition is best described in the paper.

Copy Right, IJAR, 2017, All rights reserved.

\section{Introduction:-}

The modern world has become more automated day by day, this automation makes our work easier, faster and efficient. Handwritten character recognition is the process of recognizing given characters from the large set of characters. OCR system mainly focuses on the recognition of printed or handwritten characters of a scanned images. Since the introduction of digital computer machine simulation of human reading has become a genuine evolutionary research [1]. The proficiency of any character recognition technique is specifically relied on the precision of the generated features that could exceptionally represent a character and consequently a recognition of it. Actual recognition in any OCR implementation consists of several preprocessing steps which are followed by actual recognition [2].

Optical character recognition (OCR) has been the most challenging and interesting areas from many years to the researchers. The system which can interpret and detect the text efficiently from the documents is called as optical character recognition system. OCR system has lots of practical and commercial applications like reading cheques in banks, reading envelopes, Job application form, other manuscripts in post offices, libraries, publication houses, [3,4] etc. Recognition task is difficult because of the differences in the character font types, sizes, noise in the image, etc. 
A broad research is going on in this field since few decades; handwritten character recognition is at the beginning stage in the Indian context [5].A character recognition, classification is as shown in figure 1.

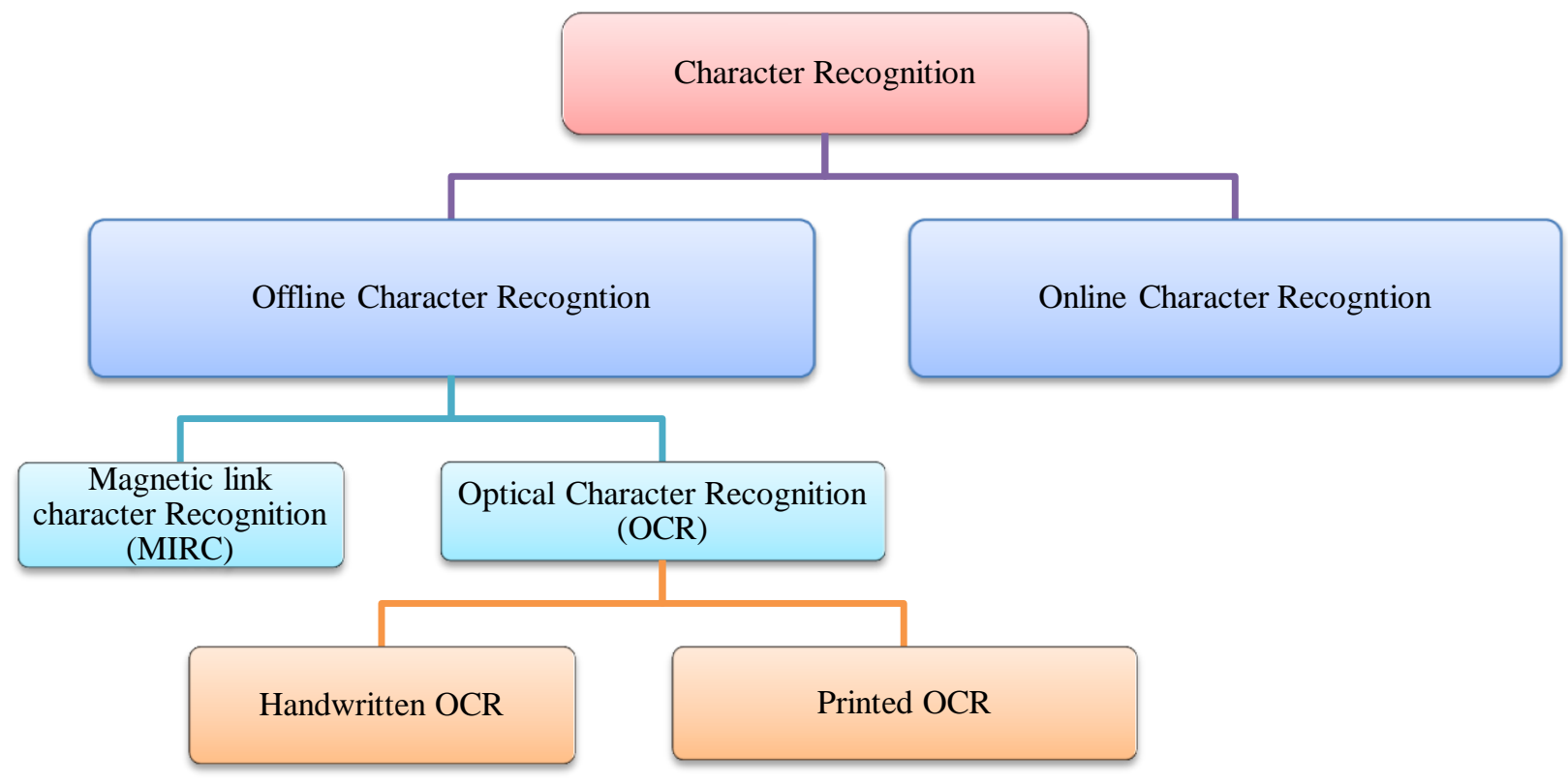

Figure 1:- Classification of Character recognition.

The procedure of reading the text components from such documents is divided into three steps:

1. Extracting the text contents or text paragraphs from the image of the document.

2. Segmentation of the lines, word, and characters from the text paragraphs.

3. Recognition of the individual's segmented characters.

In figure 2 showing the general steps followed by optical and handwritten character recognition.

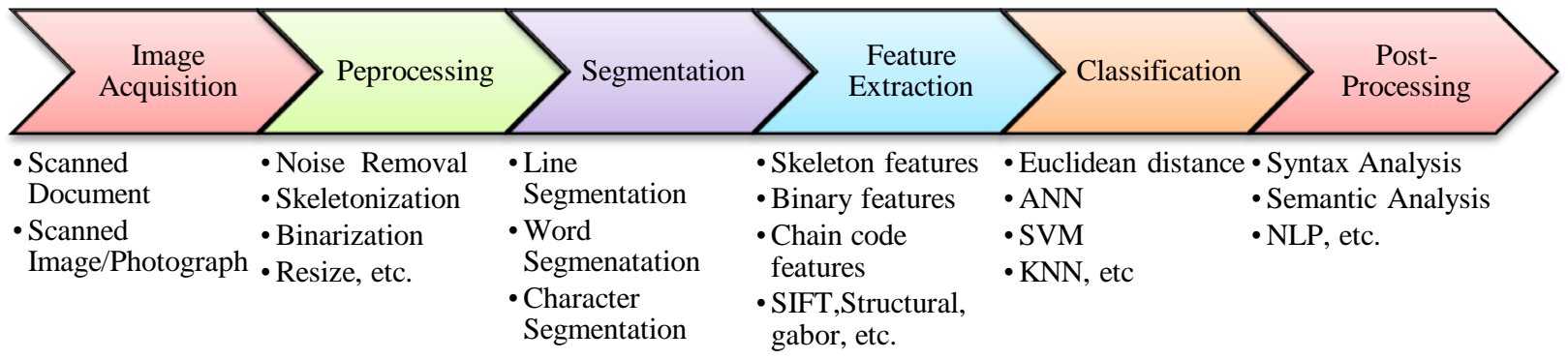

Figure 2:- General steps in printed and handwritten character recognition

From the connected character pattern to get a single order pixel skeletons is noted as skeletonization process, the process will be helpful in removal of irregularities in letters which makes the algorithm for the recognition simpler because the operation is done on character stroke of one pixel width also reduces memory required for storing and processing the input characters which definitely reduces processing time.

The need of the skeletonization algorithm in the real world is due to following reasons [6]:

a) The amount of processing data is reduced. 
b) Processing time is reduced.

c) Extraction of critical features such as endpoints, junction points, and connection among the components.

d) In pattern recognition, vectorization algorithms tasks also require skeleton image as an input.

e) On a line with a pattern, shape analysis can be easily done.

The skeletonization process is an important step in preprocessing for the printed \& handwritten document images. If the resulted skeleton is accurate, it eliminates a lot of errors in the later character recognition stage. The main challenges in thinning character images are to preserve the original shape after the characters thinning algorithm [7]. Author [8] proposed the algorithm to avoid shape distortion by detecting the ambiguous regions and avoiding the identification of medial axis points within these regions. The resultant skeleton maintains the pixel connectivity and is very close to the medial axis.by the iterative thinning process a better skeleton shape we obtained [9].

In preprocessing stage skeletonization is used for the several applications such as, script identification [10], optical character recognition OCR [11]. Writer identification [12,13], etc.

Three major skeletonization techniques are as follows:

1. Distance transformation

2. Voronoi diagram

3. Thinning

Skeletonization having the two main approaches; iterative and non-iterative [14]. As researches about both skeleton processing and adaptive binarization reach the final, conclusive state it became clear that the two preprocessing steps could be improved not only as separate entities but also as interacting stages of the document's image preparation [15]. For the later character recognition stage. The visual system in our brain can recognize printed characters and handwritten characters easily, robustly, and precisely. If the skeleton of a character is represented as a graph, the features can be more easily extracted [16].

For shape representation, skeletonization is a global space domain technique $[17,18]$, which is the most useful and important factor in Indian scripts. A large amount of literature is available for the recognition based on skeletonization in English, Arabic, and other scripts; relatively less work has been reported for the recognition of Indian languages. Reasons for this slow development is due to the cursive shape of Indian scripts, and also the large set of different patterns that exist in these languages, as opposed to English [19].

Studying the various research works has provided us with a clear insight into what the work entails, the various techniques and the subsequent complications involved. This study was conducted to review and examine the approach as extraction and classification based on the skeletonization process with a graphical approach for getting a better result [20].

This paper is organized into four sections the first section describes the introduction of the skeletonization algorithm, in Section II deals with the work related to Character recognition based on a skeletonization process for printed characters, while Section III discusses the skeletonization based recognition of handwritten Characters, Section IV reports some observations and the comparison is made for the Devanagari characters. The paper is concluded in the last section. A comprehensive bibliography of references is provided for a prospective reader. The reference includes the most relevant papers recently published as well as other papers which give a comprehensive outline of the developments in the research field of character recognition.

\section{Recognition of printed Characters:-}

From the set of learning characters and their properties, the optical character recognition for the printed characters relies on to compare from the scanned image file to the characters in the learned set. From the scan text, image document for the text recognition of the characters we have to go with different steps with the applications

In OCR, characters get recognized by pattern matching. In the case of character recognition system, especially Devanagari character recognition is more complicated as it is having multiple loops, conjuncts, upper and lower modifiers and the number of disconnected and multi-store characters [21]. 


\section{Pre-Processing:-}

As a preprocessing step, skeletonization of the character is performed using an iterative thinning algorithm based on Raster scan of the character image. In [22] a combination of structural features of the character like a number of endpoints, loops, and intersection points is calculated. Further, the thinned character image is statistically zoned into partitions, and a quadratic curve-fitting model is applied on each partition forming a feature vector of the coefficients of the optimally fitted curve. This vector is combined with the spatial distribution of the foreground pixels for each zone and hence script-independent feature representation. The approach has been evaluated experimentally on Devanagari scripts. The algorithm achieves an average recognition accuracy of 93.4\%. [23]

A new skeletonization algorithm is proposed in this article. The algorithm is combined between parallel and sequential which categorized under the iterative approach [24] Iterative techniques, the Peeling contour process iteratively parallels or sequentially; in a parallel way, the whole unwanted pixels are erased after identifying the whole wanted pixels Whereas in sequential techniques; the unwanted pixels are removed in the identifying the desired pixels in each iteration. In a non-iterative approach, the skeleton is extracted directly without examining each pixel individually, but these techniques are difficult to implement and slow as well [25]. The algorithm for thinning has been extensively tested on several Devanagari script characters [26].

To achieve the skeletonization in the pre-processing [27] following the steps:

1. Mark the start and end points.

2. Allocate deletable pixels.

3. Delete pixels that marked as deletable pixels.

4. Iteration

5. Detection of discontinuity and recovery

6. Unnecessary pixel removal

Recover pixels in an image of the finest, reliable skeleton of the image are produced in a very effective and in a simple manner.

\section{Segmentation/ Skeletonization:-}

After the preprocessing step either we can go for segmentation or the separate thinning algorithm is to be carried out to refine some pixels or reconstruction of the original shape. The pattern recognition algorithm while maintaining the configuration of the pixels and the topology of the image. Character recognition is a major research area that utilizes thinning to expedite the extraction of critical structural features of a character. Another crucial aspect of thinning is that the output should be located approximately to the medial axis of the character stroke.

The strokes of a character may consist of two regions, i.e., singular and regular regions. The intersections and junctions of the strokes belong to the singular region, while the straight and smooth parts of the strokes are categorized by regular region [28].

All traditional skeletonization algorithms are based on the symmetry analysis technique. [29] Skeleton has been defined in various ways in the different kinds of literature. All kinds of definitions result in a variety of skeletonization algorithms as well. However, it is generally agreed that a good skeleton should have some good properties [30].

Skeleton obtained from the character image from the thinning methods [6], has a wide range of applications for classification and shape analysis. Spurious stokes removal in the thinning process is a difficult problem, so [31] suggest contour based thinning method on the noisy isolated character image where skeleton gets segmented into strokes of the vector form. Which apply to the printed text and get better results compared with other thinning algorithms [32]. Skeletonization and thinning process broadly classified into two groups: raster scan-based and medial axis based [12]. [33] Proposed a parallel thinning algorithm which preserves connectivity and produces a skeleton of one-pixel thickness.

The skeleton of the cursive character shape is mathematically derived from its contour [34] [35]. In image processing, skeletonization has the wide variety of applications [36]. Digital skeletons, generated by thinning algorithms, are often used to represent objects in a binary digital image for shape analysis and classification, the skeleton is defined as a set of thin lines, arcs, and curves [37]. 
The contribution of [38] method is that it does not consider all pixels equally for performing character thinning. It is based on the substitution of pixels of strokes or curves which are the most valuable parts for character recognition, for boundary pixel removal and handling corners or junction points. There will be an indirect skeletonization method to reduce human perceptions artifacts. The method is based on analyzing regularities and singularities of shapes. A shape is first partitioned into a set of triangles using the constrained Delaunay triangulation technique. Then, regular and singular regions of the shape are identified from the partitioning. Finally, singular regions are stabilized to produce a better result. Experiments show that skeletons obtained from the proposed method closely resemble human perceptions of the underlying shapes [29,39].

Some algorithms suffer from these traditional problems such as the one-pixel width of the skeleton and skeleton connectivity as well. Distortion in the topology of shape skeleton is a serious problem in the thinning application [40]. Whereas, several techniques are failing to preserve the shape topology [41]. Spurious tails and rotating the text shape is another serious problem and most thinning methods are failing [42].

An essential challenge for computing the primary skeleton is that it is difficult to decide the symmetric pairs from the boundaries of the character strokes in both continuous and discrete domains [28]. The time consumed in the skeleton extraction step may be ignored compared with the contour detection. Therefore, the implementation of the proposed algorithm is expected to be easy, direct and fast [43].

\section{Feature Extraction:-}

Extracting several features from document text such as a horizontal line, vertical line, right slant line, left slant line, curve and holes from the skeleton image projection can be used as a feature extraction technique [44], wherein the direction of projection can be the horizontal axis, vertical axis, the diagonal axis or a combination of all three [45]. The features that can be extracted from a grayscale image can be classified into two categories: structural features and frequency features. The structural features include direction features, skeleton feature, topological feature, etc. Frequency features are very effective for the recognition of low-resolution gray characters such as Fourier transform and wavelet transform $[46,47]$.

An important aspect of image analysis is feature extraction prior to the classification. Certain characteristics are used to describe a character; these characters contain a large volume of data in the form of pixel intensity values [30]. The data or the sets of the characters collected would generate huge amounts of data which might vastly be redundant and repetitive. Reducing the amount of information and being able to identify the information involves the use of the characteristic features of individual data, to be more precise, a feature vector [48]. Identifying these features involves several feature extraction techniques. The techniques used and the features to be extracted should be carefully chosen to extract the relevant information required for the subsequent steps of identifying the characters using several algorithms [49].

Article [50] proposes a model of an efficient feature set extraction technique using statistical and structural features of the text image for the script-independent character recognition (tested on English and Urdu also). Results generated by the experiments on the tested images conform to the greater level of efficiency with respect to the size of the feature set, the techniques used and the features to be extracted should be carefully chosen to extract the relevant information required for the subsequent steps of identifying the characters using several algorithms.

Chain code histogram features are extracted from contour points by chain coding of the scaled character bitmapped image and moment-based features are extracted from scaled, thinned one-pixel wide skeleton of the character image. Two kinds of eigenvectors, namely wavelet energy feature and wavelet energy proportion feature were used by [51] In their work, they used features like aspect ratio, end points, junction, loop, contour direction, moment feature vector [52]. Shadow features of a character were extracted by [53]. The Shadow is basically the length of the projection on the sides.

From the survey, we observed the errors in extracting the features for printed Devanagari characters because of incorrect character segmentation of touching or broken characters and due to the upper and lower modifiers of Devanagari text, [1] skeletal features tended to give the better features or a technique which gives the strong result in the printed Devanagari character recognition. 


\section{Classification:-}

The character image can be expressed in the form of a vector of smallest length, which is called a feature vector. For recognition of many variations in the font sizes of the same character, features that are invariant to certain change on the characters need to be used. The segmentation of individual characters can itself provides estimates [54] of size and location, but the feature extraction method may often provide more accurate estimates. According to the features of each character, it gets divided into different classes [55].

The total number of nodes in the tree is computed and the features are computed for each node that is visited and then, based on these computations the characters are classified and recognized [56].

A fair comparison of classifiers is difficult because of many classifiers, especially neural networks, are flexible in implementation and their performance is affected by human factors [3]. In the character recognition field, the comparison of methods is more difficult because many processing steps (pre-processing, feature extraction, classification) are involved. Even on experiments using the same training and test data, researchers often compare the performance at the system level: the final recognition rate by integrating all techniques. It is hard to decide what method at which step is the most influential [57].

Many techniques [58], e.g., nonlinear normalization and direction feature extraction, are variable in implementation details. Hence, we suggest opening source codes of standard techniques for every processing step of character recognition, such that researchers can fairly compare the methods of a special step. For benchmarking classification methods, we can alternatively release standard feature data other than image data.

\section{Recognition:-}

In recognition of character it is compared with the stored database if features get a match then that character gets recognized. Devanagari character recognition to classify an unknown character into one of the known classes of character involves extraction of features by some definable characteristics $[59,60]$.

Recognition of characters from hypothesized strokes has been accomplished by [61] The hypothesized strokes are subjected to a proximity analysis. This returns a set of strokes which comprise a character. A one-dimensional discrete multiwavelet transform has been applied to a previously extracted contour of the character. Invariant moments and Zernike moments that have been progressively used in pattern recognition were used to extract features of printed Kannada characters by [62].

In the recognition stage only we can decide which features or classifiers is better for the language detection or extraction accuracy.

\section{Recognition of Handwritten Characters:-}

Since writing styles of people vary, it has becomes practically difficult for the computer to recognize the handwritten characters. The several factors influence the recognition rate like poor quality of the character/document, the typical writing style of different writers, similar shape characters etc. that makes the feature extraction process tedious [63]. To fulfill this task, handwritten character recognition comes into the picture.

\section{Pre-Processing:-}

Major research works carried out in pre-processing techniques, applied on character images of the skeletonization process for handwritten characters are discussed in this section. In pre-processing, one normally performs thresholding (binarization), size, normalization, morphological, skeletonizing (thinning), skew correction and slant normalization. In the case of isolated handwritten character images, if the samples are collected in separate boxes provided for writing, the skew and slant variations present are limited to a tolerable range. In such cases, skew correction and slant normalization are not relevant. So only the foremost pre-processing operations, namely thresholding, size normalization and thinning are reviewed in this instance because of its central role in preprocessing of data images, the design of thinning or skeletonization algorithms has been a very active research area since its inception. Thinning procedure transforms digital binary patterns into connected skeletons of unit width. Many of the handwritten character recognition works reported are based on thinned characters [64-75]. Integrated Circuits have also been designed for this purpose [76].

One of the experiments on thinning of character patterns was conducted by [77]. In his work, an averaging operation over a square window with a high threshold is used for thinning the input image. Kirsch et al., [78] proposes a 
cluster operation to obtain a thin line representation of character patterns. There are two basic approaches for thinning a digital pattern: iterative boundary removal and distance transformation the former method is widely used for handwritten character images since it removes boundary pixels of a connected component that neither preserve the connectivity of the pattern nor represent any of its significant geometrical features. Two basic implementation techniques have been used for this operation. One is the sequential approach, in which pixels are examined and transformed depending on the previously processed results. The other is a parallel algorithm, which operates on all pixels simultaneously [79].

In the work [80] have proposed a novel rule-based algorithm for thinning. The important characteristic of this algorithm is that it thins symbols to their central lines. So, the shape of the symbol is preserved and it is rotation invariant in nature. Results by [81] of thinning performed on English, Chinese and Arabic characters and different geometrical shapes disclose that this algorithm is very efficient in preserving the topology of symbols and letters written in any language.

In summary, the research findings reported so far on pre-processing techniques are good enough to deal with the printed or neatly written document images. The invention of effective and robust application-specific pre-processing techniques, capable of handling degraded and unconstrained handwritten document images, is to be seriously addressed.

\section{Segmentation/Skeletonization:-}

Skeleton-based features work on the word and characters of the skeleton image which is obtained by thinning the binary raster representation of the word image or character image [83]. GSC features were first proposed by [82] and were designed to work with the binarized images. A bounding box is placed around the image and the features are computed. The feature maps are sampled by placing a $4 \times 4$ grid on the maps. The features themselves are computed independently of this sampling grid. The GSC features approximate a multi-resolution approach by being generated at three ranges: local, intermediate and global. The gradient features detect local features of the image and provide a great deal of information about the stroke shape within a short neighborhood [84].

To improve the sequential thinning procedure an optimized homeotropic transformation of the structural elements. [15] Producing less coarse skeleton lines with a reduced number of thinning cycles.

The skeleton of a character embodies the basic shape information which is, in a sense, font independent. Extracting high-quality skeleton for skeleton matching and topological analysis is, therefore, an ideal method for character recognition, whereas in practice it remains a dream of many technicians, because of its subjective character and hard to avoid deformations. Plenty of reports about this research in past years can be found [85].

\section{Feature Extraction:-}

Preprocessing and Feature Extraction is very important steps in automatic cursive handwritten word recognition. Three different feature extraction methods are presented, the Sliding Window with Local feature extraction method uses only the original image and the baseline information, without any others preprocessing. We achieved recognition rates of up to $89 \%$ on word level using the skeleton based method for baseline estimation and skeleton direction features. [86]

Although, many pieces of work have been done for the recognition of printed characters in Indian languages, only a few attempts have been made towards the recognition of handwritten characters [87-89] have presented a Devanagari numeral recognition system in which the presence or absence of four basic primitives, namely, horizontal and vertical line segments, right and left slant, and their interconnections are used for the recognition with a decision tree.

Legendre moments for handwritten Arabic numerals provide informative feature extraction in this work. Feature extraction was performed by Fourier-modified direct Mellin transforms (FMDMT) and Zernike moments by [90] to make the pattern translation scale, and rotation invariant. Rotation invariant is obtained by Zernike moments which are the mapping of the image into a set of complex orthogonal polynomials. In the work on script identification in Indian documents [91], the feature extractor comprises two stages. In the first stage, the document image is dilated using $3 \times 3$ masks in horizontal, vertical, right, diagonal, and left diagonal directions. In the next stage, average pixel distribution is found in these resulting images. 
The extracted character features used in the self-organization matching are center loci, orientation, and major axes of ellipses which fit the inked area of the patterns. Simulations provide encouraging results using the proposed method [92].

The Centralized moments technique was used for feature extraction wherein a set of seven moment invariant features of the characters is extracted after the segmentation stage for each segmented character [93]. The features such as curvature and slope of the stroke, axis ratio, endpoint sequence, etc., which uniquely represent the stroke, can be used for the identification of handwritten characters [94]. The recognition system developed with these features was independent of the language.

The characters feature extraction has the number of techniques accordingly many features can be extracted to form the feature vector. From the skeleton of the image as listed in $[95,96]$.

\section{Classification:-}

Classification stage is to recognize characters or words. After features that represent the raw input data are extracted, classification stage should use the data to recognize the feature class based on the properties of the features. There are many techniques available in features. There are many techniques, many techniques available in the classification method that can be applied in the handwritten character recognition process. The classification method can have traced from template matching, statistical approach, syntactic, and Neural Network [98].

A decision tree is to employ for the analysis of hand printed Devanagari characters or Numerals use a distinct MLP classifier, each classifier either classifies or rejects an input numeral at a coarser resolution level [97].

Classification is done prior to recognition where the handwritten Devanagari characters are classified into three major categories, namely end-bar characters, middle-bar characters and characters and characters without any bar based on the presence of a vertical bar. The recognition of handwritten characters in is based on the modified exponential membership function fitted to the fuzzy sets derived from the features of the characters. A Reuse Policy policies are also utilized to improve the speed of the learning process

\section{Recognition:-}

In a recent research work [99], proposed a new edge adaptive algorithm for zooming digital images. In their work, the character image is binarized and the height and width of the character pattern found out. The original grayscale image is then cropped into the minimum rectangle enclosing the character curve have the transformation and Bilinear Interpolation algorithm [100]. All the above studies indicate that size normalization of handwritten character images simplifies and enhances the performance of recognition systems.

The system shown in figure 3 recognizes isolated handwritten character patterns. The character patterns are written within formatted boxes to simplify the segmentation. Grammar rules of the addresses are incorporated into the recognition process to improve the accuracy and reduce the search, characters which can be classified. 


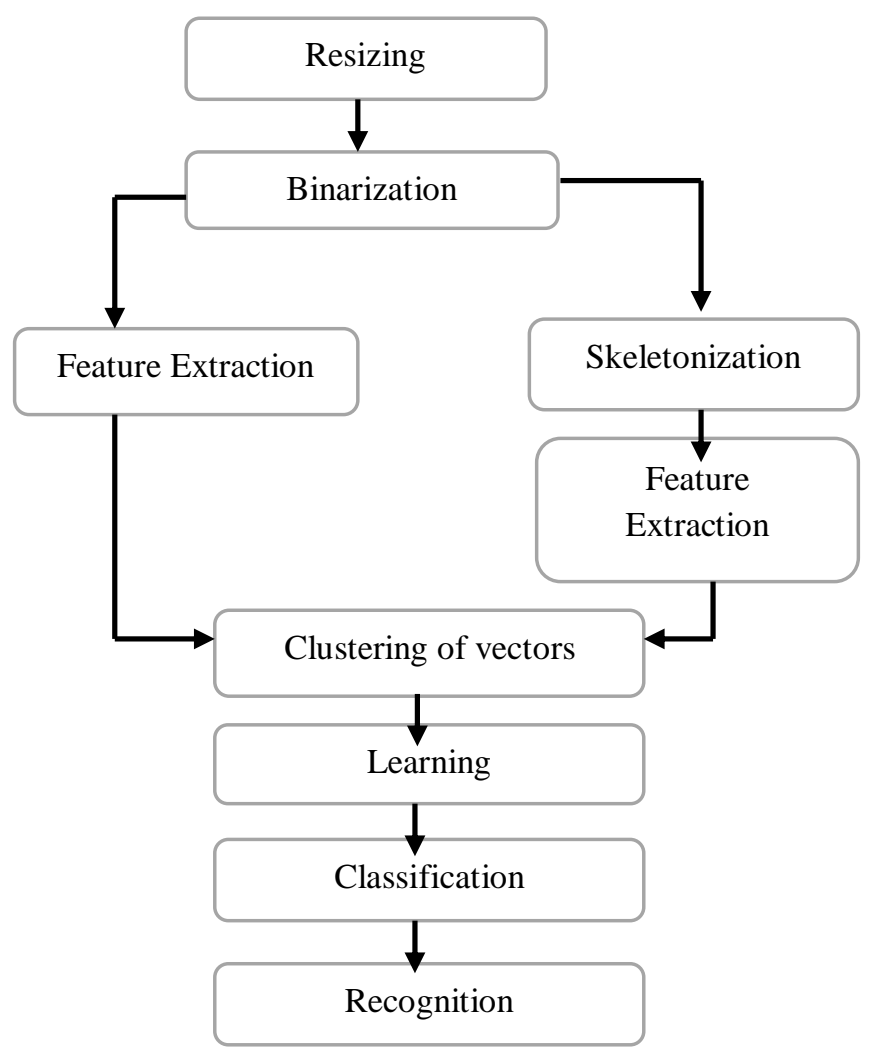

Figure 3:- Recognition Steps/Process.

\section{Observation and Results:-}

In the development, optical character recognition here reviewed the introductory concepts and stages to identify input characters in the image correctly being analyzed many automated process systems [101]. Variations in the skeleton based algorithms, the choice of algorithm for an application has turned out to be exceptionally troublesome, and a researcher in this area is often confronted which algorithm to be used. For this reason, we observed and compare the performance of different skeletonization algorithms and to examine the effects based on real-life data. Best suited algorithm having the significance and representation of the different mode operation of skeletonization Method [102].

From the study [103] Observed that the skeletonization system has the following features.

a) Symmetries analysis as an origin a skeleton point directly can be computed with the detection of the contour line, instead of indirect computing of an inscribed circle or symmetric triangle which having the immediate and straightforward approach. In addition to this, its computation cost is also relatively low.

b) Skeleton obtained by the prevailing algorithms marginally deviates from the center. In a mathematical sense, the mined skeleton is placed inside the underlying strokes.

c) Character stroke obtained using wavelet-based amendment processing to retain the sufficient information of original characters to get the skeleton in the singular region.

d) A few techniques can process a shape with holes and various shapes, which is applicable for the binary and gray level images. The problem occurs in automatically selecting the scale of the wavelet to transform according to the width of the character strokes.

How to choose the denoising threshold are currently under investigation. [28]

We observed that Skeletonization is a having a huge stride in many image processing applications for few decades. Skeletonization of digitized images is needed due to reasons described in [104]. 
The Effective skeletonization algorithm reduces the image into the thin like objects which will also retain the topological and geometric properties. However, a good skeletonization algorithm must have the following features [18].

1. The resulting skeletons should maintain connectivity.

2. The resulting skeletons should be of a unit pixel width.

3. No excessive deletion of pixels should take place.

4. It should perform better in terms of execution time.

5. It should ideally compress the data.

The process of skeletonization is an important step in preprocessing printed \& handwritten documents' images. If the resulted skeleton is accurate, it eliminates a lot of errors in the later character recognition stage. Pros and cons of the different skeletonization algorithm are shown in Table 1.

Table 1:- Advantages and disadnatages of different skeletonization algorithm.

\begin{tabular}{|c|c|c|}
\hline Sr. No. & Advantages / Disadvantages & Methods \\
\hline 1. & $\begin{array}{l}\text { Advantage: } \\
\text { a. Parallel speed is superior. } \\
\text { b. Produces very thin medial curves. } \\
\text { Disadvantage: } \\
\text { Produces noisy branches in the skeleton }\end{array}$ & $\begin{array}{l}\text { Guo } \\
{[105]}\end{array}$ \\
\hline 2. & $\begin{array}{l}\text { Advantage: } \\
\text { a. Preserves connectivity } \\
\text { b. Contour noise immunity. } \\
\text { c. Efficient in terms of execution time. } \\
\text { Disadvantage: } \\
\text { The resulting skeletons are not of unitary thickness }\end{array}$ & $\begin{array}{l}\text { Zhang T et. al. } \\
{[106]}\end{array}$ \\
\hline 3. & $\begin{array}{l}\text { Advantage: } \\
\text { a. Fast } \\
\text { b. Reliable } \\
\text { c. High immunity to boundary noise. } \\
\text { Disadvantage: } \\
\text { It takes more computation time. }\end{array}$ & $\begin{array}{l}\text { Zhou } \mathrm{R} \text { et.al. } \\
{[107]}\end{array}$ \\
\hline 4. & $\begin{array}{l}\text { Advantage: } \\
\text { a. Effective } \\
\text { b. Fast } \\
\text { c. Can thin any symbol in any language, irrespective of the direction of rotation. } \\
\text { Disadvantage: } \\
\text { Unable to thin two-pixel width lines. }\end{array}$ & $\begin{array}{l}\text { Ahmed M et.al. } \\
{[108]}\end{array}$ \\
\hline 5. & $\begin{array}{l}\text { Advantage: } \\
\text { a. No excessive erosion } \\
\text { b. Produce thin skeletons. } \\
\text { Disadvantage: } \\
\text { Ran } 18 \% \text { slower than A-W algorithm, more execution time. }\end{array}$ & $\begin{array}{l}\text { Rockett P et.al. } \\
{[109]}\end{array}$ \\
\hline 6. & $\begin{array}{l}\text { Advantage: } \\
\text { a. Z-S algorithm preserves connectivity of the skeletons. } \\
\text { b. Shows better results in Noise sensitivity measurements. } \\
\text { Disadvantage: } \\
\text { Thinning rate i.e. Calculated is not good for vectorization of roads. }\end{array}$ & $\begin{array}{l}\text { Tarabek P et.al. } \\
{[110]}\end{array}$ \\
\hline 7. & $\begin{array}{l}\text { Advantage: } \\
\text { a. Preserve the connectivity } \\
\text { b. Produce thin skeletons. } \\
\text { Disadvantage: } \\
\text { Edge based iterative thinning algorithm is time taken as } \\
\text { compared to optimized thinning algorithm. }\end{array}$ & $\begin{array}{l}\text { Padole G et.al. } \\
\text { [111] }\end{array}$ \\
\hline
\end{tabular}




\begin{tabular}{|l|l|l|}
\hline 8. & $\begin{array}{l}\text { Advantage: } \\
\text { a. It produces a unit-pixel-wide skeleton. } \\
\text { b. Better connectivity in output skeletons. } \\
\text { Disadvantage: } \\
\text { As K3M is iterative thinning algorithm, so it requires much more computing } \\
\text { power than other algorithms. }\end{array}$ & $\begin{array}{l}\text { Saeed K. et. al. } \\
{[25]}\end{array}$ \\
\hline 9. & $\begin{array}{l}\text { Advantage: Performance is high in terms of execution time. } \\
\text { Disadvantage: Topology problem. Cannot preserve shape sometimes. }\end{array}$ & $\begin{array}{l}\text { Abu-Ain } \\
\text { et.al. [6] }\end{array}$ \\
\hline 10. & $\begin{array}{l}\text { Advantage: } \\
\text { a. Skeletons are perfectly 8-connected } \\
\text { b. Does not result in excessive erosion } \\
\text { c. Produces more quality images } \\
\text { Disadvantage: Not efficient in terms of execution time. }\end{array}$ & $\begin{array}{l}\text { Jagna A et.al. } \\
{[112]}\end{array}$ \\
\hline 11. & $\begin{array}{l}\text { Advantage: } \\
\text { a. One-pixel wide skeleton } \\
\text { b. No excessive erosion. } \\
\text { Disadvantage: } \\
\text { Requires a number of iterations. }\end{array}$ & $\begin{array}{l}\text { Kwon J. et.al. } \\
{[113]}\end{array}$ \\
\hline
\end{tabular}

Comparison of the skeletonization techniques according to an approximation for the true skeleton compiled with topological and geometrical (translation, rotation, and scaling) is shown in Table 2.

Table 2:- Comparison of skeletonization techniques.

\begin{tabular}{|c|c|c|}
\hline Topological & Geometrical & Methods \\
\hline No & Yes & Distance transform \\
\hline Yes & Yes & Voronoi-skeleton \\
\hline Yes & No & Thinning \\
\hline
\end{tabular}

For Devanagari Characters or words or Numerals, some good results studied from different papers which having the skeletonization process or step is shown in Table 3

Table 3:- Survey of Devnagari characters /words/Numerals recognition techniques

\begin{tabular}{|l|l|l|l|l|l|}
\hline Sr.No. & Method & Feature & Classifier & Dataset & Accuracy (in \%) \\
\hline 1. & Sharma et.al [114] & Chain code & Quadratic & 11,270 & 80.36 \\
\hline 2. & Deshpande et.al. [115] & Chain code & RE \& MED & 5,000 & 82 \\
\hline 3. & Govindraju et.al. [116] & Gradient & NN & 4506 & 84 \\
\hline 4. & Arora et.al. [117] & Structural & FFNN & 50,000 & 89.12 \\
\hline 5. & Bajaj et.al. [118] & $\begin{array}{l}\text { Density moment } \\
\text { \& Descriptive }\end{array}$ & $\begin{array}{l}\text { Multiclassifier } \\
\text { connectionist }\end{array}$ & $\begin{array}{l}\text { Not } \\
\text { Specified }\end{array}$ & 89.68 \\
\hline 6. & Hanmandlu et.al. [119] & Vector Distance & Fuzzy sets & 4750 & 90.65 \\
\hline 7. & Arora et.al. [120] & Shadow \&CH & MLP \& MED & 7154 & 90.74 \\
\hline 8. & Kumar et.al. [121] & Gradient & SVM & 25,000 & 94.1 \\
\hline 9. & Pal et.al. [122] & $\begin{array}{l}\text { Gradient } \\
\text { Gaussian Filter }\end{array}$ & Quadratic & 36,172 & 94.24 \\
\hline 10. & Pal et.al. [123] & Gradient & SVM \&MODF & 36,172 & 95.13 \\
\hline 11. & Mahesh et.al. [124] & CGNL & SVM & 36,172 & 96.03 \\
\hline 12. & Shushma et.al. [125] & $\begin{array}{l}\text { Wavelet } \\
\text { Transform }\end{array}$ & NN & $\begin{array}{l}\text { Not } \\
\text { Specified }\end{array}$ & 96.23 \\
\hline 14. & Sharma et. al. [126] & Chain code & Quadratic & 22,556 & 98.86 \\
\hline 15. & Pal et. al. [127] & Gradient & MQDF & 23,340 & 98.41 \\
\hline 16. & $\begin{array}{l}\text { Bhattacharya } \\
\text { Chaudhuri et. al.[128] }\end{array}$ & Wavelet & MLP & 22.556 & 99.27 \\
\hline
\end{tabular}

In summary, the research findings reported so far on pre-processing techniques are good enough to deal with the printed or neatly written document images. The invention of effective and robust application-specific pre-processing 
techniques, capable of handling degraded and unconstrained handwritten document images, is to be seriously addressed.

\section{Conclusion and future work:-}

In this Paper, a recent study on character recognition based on skeletonization techniques or process for the printed as well as for the handwritten characters. We concluded the point that skeletonization method would better choice to reduce the processing or recognition time, Research work published for the printed and handwritten characters is well described and the best suitable method for the Devanagari character recognition is explained in the paper. We can also develop character classification method using a similar skeleton graph for the similar character in printed while in handwritten that may be different how they are extracted is also explained.

This paper shows the performance comparison table of a different technique that was proposed for the text detection and extraction of the characters from an image. There having several approaches which have their own pros and cons, apart from this there will be several methods for the skeletonization process, no single specified method that fits for all the applications.

In future studies, we can implement some new algorithm for the Devanagari characters by the study of this skeletal feature a better recognition performance can be obtained. Text extraction from an image with better accuracy within the short time for the regional language is the main motive.

\section{References:-}

1. Jayadevan, R.et al. "Offline recognition of Devanagari script: A survey."IEEE Transactions on Systems, Man, and Cybernetics, Part C (Applications and Reviews) 41.6 (2011): 782-796.

2. Rakesh, Gupta, and Kaur Rajpreet, "Skeletonization Algorithm for Numeral Patterns", International Journal of Signal Processing, Image Processing and Pattern Recognition 1.1 (2008): 63-72.

3. U. Pal and B. B. Chaudhuri, "Indian script character recognition: A Survey", Pattern Recognition, Vol. 37, pp. 1887-1899, 2004.

4. L. Liu and C. Y. Suen, "A new benchmark on the recognition of handwritten Bangla and Farsi numeral characters", In Proc. 11th ICFHR, 2008.

5. Shivangkumar R Patel, Ms. Jasmine Jha, "Handwritten Character Recognition using Machine Learning Approach - A Survey", (EESCO) - 2015 IEEE.

6. Abu-Ain, Waleed, et al. "Skeletonization Algorithm for Binary Images." Procedia Technology 11(2013):704709.

7. Bag, Soumen, and Gaurav Harit. "Topographic Feature Extraction for Bengali and Hindi Character Images." arXiv preprint arXiv:1107.2723 (2011).

8. Wei, C., S. Lichun, et al., Improved Zhang-Suen thinning algorithm in binary line drawing applications. Systems and Informatics (ICSAI), 2012 International Conference on, 2012.

9. Bag, Soumen, and Gaurav Harit. "An improved contour-based thinning method for character images." Pattern Recognition Letters 32.14 (2011): 1836-1842.

10. Gopakumar, R.., Subbareddy, N.V., Makkithaya, K, Acharya, U. D., Script Identification from Multilingual Indian Documents using Structural Features. Journal of Computing, 2010: 2: 106-111. 709.

11. Ali, M. A., An efficient thinning algorithm for Arabic ocr systems. Signal \& Image Processing-An International Journal (SIPIJ), 2012: 3: 31-38.

12. Abu-Ain, T. A. H., W. A. H. Abu-Ain, et al., Offline Arabic Character-Based Writer Identification - a Survey, in International Journal of Advanced Science, Engineering and Information Technology, Proceeding of the International Conference on Advanced Science, Engineering and Information Technology Bangi, Malaysia, 2011.

13. Bataineh, B., Abdullah, S. N. H. S., Omar, K., An adaptive local binarization method for document images based on a novel thresholding method and dynamic windows. Pattern Recognition Letters, 2011: 32: 18051813.

14. Nemeth, G., K. Palagyi., Topology preserving parallel thinning algorithm. International Journal of Imaging System and Technology, 2011: 21: 37-44.

15. LACRĂMA, DANL, and Florin Alexa. "Improved structuring element for handwriting and hand printed characters skeleton." transformation (especially scale) 3 (2010): 8. 
16. Huang, Yea-Shaun. The combination of multiple classifiers for the recognition of totally unconstrained handwritten numerals. Diss. Concordia University, 1994.

17. Dr.V.Vijaya Kumar, A.Srikrishna, Sadiq Ali Shaik, S. Trinath, A New Skeletonization Method Based on Connected Component Approach, IJCSNS International Journal of Computer Science and Network Security, VOL.8 No.2, February 2008.

18. Meghwal, Ashwani Kumar, et al. "A simple and efficient non-pixel based skeletonization method using direction model." (2006): 526-532.

19. Kannan, R. Jagadeesh, and R. Prabhakar. "A comparative study of optical character recognition for Tamil script." European Journal of Scientific Research 35.4 (2009): 570-582.

20. Mori, Shunji, Ching Y. Suen, and Kazuhiko Yamamoto. "Historical review of OCR research and development." Proceedings of the IEEE 80.7 (1992): 1029-1058.

21. Arora, Sandhya, et al. "Combining multiple feature extraction techniques for handwritten devnagari character recognition."Industrial and Information Systems, 2008. ICIIS 2008. IEEE Region 10 and the Third International Conference on. IEEE, 2008.

22. Khanduja, Deepti, and Neeta Nain. "Script independent feature set for handwritten text recognition." Information and Communication Technology, Electronics and Microelectronics (MIPRO), 2014 37th International Convention on. IEEE, 2014.

23. L. Lam, S.W. Lee and C.Y. Suen. "Thinning Methodologies -A comprehensive survey." IEEE Transactions on Pattern Analysis and Machine Intelligence, Vol.14, pp.869-885, 1992.

24. Fujita, Kazuhisa. "Extract an essential skeleton of a character as a graph from a character image." arXiv preprint arXiv: 1506.05068(2015).

25. Saeed, K., Tabedzki, M., Rybnik, M., Adamski, M., K3M: A universal algorithm for image skeletonization and a review of thinning techniques. Applied Mathematics and Computer Science, 2010: 20, 317-335.

26. Rajashekararadhya, S. V., and P. Vanaja Ranjan. "Efficient zone-based feature extraction algorithm for handwritten numeral recognition of four popular South Indian scripts." Journal of Theoretical \& Applied Information Technology 4.12 (2008).

27. Ali, M., and Kasmiran Bin Jumari. "Skeletonization Algorithm for an Arabic handwriting." WSEAS Transactions on Computers 2.3 (2003): 662-667.

28. You, Xinge, and Yuan Yan Tang. "Wavelet-based approach to character skeleton." IEEE Transactions on Image Processing 16.5 (2007): 1220-1231.

29. Zou, Ju Jia, and Hong Yan. "Skeletonization of ribbon-like shapes based on regularity and singularity analyses." IEEE Transaction on Systems, Man, and Cybernetics, Part B(Cybernetics) 31.3(2001): 401-407.

30. Lee, Seong-Whan, Louisa Lam, and Ching Y. Suen. "A systematic evaluation of skeletonization algorithms." International Journal of Pattern Recognition and Artificial Intelligence 7.05 (1993): 1203-1225.

31. L. Lam, S.W. Lee and C.Y. Suen. "Thinning Methodologies -A comprehensive survey." IEEE Transactions on Pattern Analysis and Machine Intelligence, Vol.14, pp.869-885, 1992.

32. Goswami, Mukesh M., and Suman K. Mitra. "Offline handwritten Gujarati numeral recognition using low-level strokes." International Journal of Applied Pattern Recognition 2.4 (2015): 353-379.

33. Datta, Amitava, and Swapan K. Parui. "A robust parallel thinning algorithm for binary images." Pattern Recognition 27.9 (1994): 1181-1192.

34. Di Ruberto, Cecilia. "Recognition of shapes by attributed skeletal graphs." Pattern Recognition 37.1(2004): 21 31.

35. Zou, Ju Jia, and Hong Yan. "Skeletonization of ribbon-like shapes based on regularity and singularity analyses." IEEE Transaction on Systems, Man, and Cybernetics, Part B(Cybernetics) 31.3(2001): 401-407.

36. Kong, T. Yung, and Azriel Rosenfeld, eds. Topological algorithms for digital image processing. Vol. 19. Elsevier, 1996.

37. Jang, B-K., and Roland T. Chin. "Analysis of thinning algorithms using mathematical morphology." IEEE Transactions on pattern analysis and machine intelligence 12.6 (1990): 541-551.

38. Zhu, Xuefang, and Shuyi Zhang. "A shape-adaptive thinning method for binary images." Cyberworlds, 2008 International Conference on. IEEE, 2008.

39. Russ, John C. The image processing handbook. CRC Press, 2016.

40. Abu-Ain, Waleed, Siti Norul Huda Sheikh Abdullah, and Khairuddin Omar. "a simple iterative thinning algorithm for text and shape binary images." Journal of Theoretical \& Applied Information Technology 63.2 (2014).

41. Cohen-Steiner, David, Pierre Alliez, and Mathieu Desbrun. "Variational shape approximation." ACM Transactions on Graphics (TOG). Vol. 23. No. 3. ACM, 2004. 
42. Bansal, Preeti, and Balwinder Kaur. "To propose an improvement in Zhang-Suen Algorithm for Image Thinning in DIP." International Journal of Computer Science and Information Security 14.5 (2016): 205.

43. Fouladi, Kazim, Babak N. Araabi, and Ehsanollah Kabir. "A fast and accurate contour-based method for writerdependent offline handwritten Farsi/Arabic subwords recognition." International Journal on Document Analysis and Recognition (IJDAR) 17.2 (2014): 181-203.

44. Khawaja, Attaullah, et al. "Recognition of printed Chinese characters by using Neural Network." Multitopic Conference, 2006. INMIC'06. IEEE. IEEE, 2006.

45. Rosser, Roy J., et al. "Image insertion in video streams using a combination of physical sensors and pattern recognition." U.S. Patent No. 6,100,925. 8 Aug. 2000.

46. Reed, Todd R., and JM Hans Dubuf. "A review of recent texture segmentation and feature extraction techniques." CVGIP: Image Understanding 57.3 (1993): 359-372.

47. Liu, Cheng-Lin, and Xiang-Dong Zhou. "Online Japanese character recognition using trajectory-based normalization and direction feature extraction." Tenth International Workshop on Frontiers in Handwriting Recognition. Suvisoft, 2006.

48. Lang, Andrew K., and Donald M. Kosak. "Information system and method for filtering a massive flow of information entities to meet user information classification needs." U.S. Patent No. 5,867,799. 2 Feb. 1999.

49. Pesaresi, Martino, Vasileios Syrris, and Andreea Julea. "A new method for earth observation data analytics based on symbolic machine learning." Remote Sensing 8.5 (2016): 399.

50. Lorigo, Liana M., and Venugopal Govindaraju. "Offline Arabic Handwriting recognition: a survey." IEEE transactions on pattern analysis and machine intelligence 28.5 (2006): 712-724.

51. Guo, Hai, and Jing-ying Zhao. "Segmentation method for Naxi pictograph character recognition." Journal of Convergence Information Technology 5.6 (2010): 87-98.

52. Liu, Cheng-Lin, and Yue Lu, eds. Advances in Chinese Document and Text Processing. Vol. 2. World Scientific, 2017.

53. Arora, Sandhya, et al. "Performance comparison of SVM and ANN for handwritten devnagari character recognition." arXiv preprint arXiv:1006.5902 (2010).

54. Trier, Øivind Due, Anil K. Jain, and Torfinn Taxt. "Feature extraction methods for character recognition-a survey." Pattern recognition 29.4 (1996): 641-662.

55. Chacko, Anitha Mary MO, and KS Anil Kumar. "Offline Malayalam Character Recognition: A Comparative Study Using Multiple Classifier Combination Techniques." Information Systems Design and Intelligent Applications. Springer India, 2016. 69-77.

56. Keysers, Daniel, et al. "Multi-language online handwriting recognition." IEEE transactions on pattern analysis and machine intelligence 39.6 (2017): 1180-1194.

57. Liu, Cheng-Lin, and Hiromichi Fujisawa. "Classification and learning methods for character recognition: Advances and remaining problems." Machine learning in document analysis and recognition. Springer Berlin Heidelberg, 2008. 139-161.

58. Arica, Nafiz, and Fatos T. Yarman-Vural. "An overview of character recognition focused on off-line handwriting." IEEE Transactions on Systems, Man, and Cybernetics, Part C (Applications and Reviews) 31.2 (2001): 216-233.

59. Arora, Sandhya, et al. "study of different features on handeritten Devnagari character."2009 Second International conference on Emerging Trends in Engineering \& Technology, IEEE, 2009.

60. Selvakumar Raja, S., and Mala John. "A Novel Method of Tamil Character Recognition Using Slope Method." IUP Journal of Science \& Technology 6.2 (2010).

61. Jayaraman, Anitha, C. Chandra Sekhar, and V. Srinivasa Chakravarthy. "Modular approach to recognition of strokes in Telugu script." Document Analysis and Recognition, 2007. ICDAR 2007. Ninth International Conference on. Vol. 1. IEEE, 2007.

62. Kunte, R. Sanjeev, and R. D. Samuel. "A simple and efficient optical character recognition system for basic symbols in printed Kannada text." Sadhana 32.5 (2007).

63. Jangid, Mahesh, and Sumit Srivastava. "Gradient Local Auto-Correlation for handwritten Devanagari character recognition." High Performance Computing and Applications (ICHPCA), 2014 International Conference on. IEEE, 2014.

64. Sherman, H., et al. "A figure of merit for catheter sampling systems." Circulation research 7.3 (1959): $303-313$.

65. Deutsch, E. S. "Preprocessing for character recognition." Proceedings of the IEE-NPL Conference on Pattern Recognition. 1968.

66. Alcorn, T. M., and C. W. Hoggar. "Preprocessing of data for character recognition." Marconi Review 32 (1969): 61-81. 
67. Triendl, Ernst E. "Skeletonization of noisy handdrawn symbols using parallel operations." Pattern Recognition 2.3 (1970): 215-226.

68. Beun, M. "FLEXIBLE METHOD FOR AUTOMATIC READING OF HANDWRITTEN NUMERALS. 1. GENERAL DESCRIPTION OF RECOGNITION METHOD." Philips Technical Review 33.4 (1973): 89-101.

69. Tamura, Hideyuki, Shunji Mori, and Takashi Yamawaki. "Textural features corresponding to visual perception." IEEE Transactions on Systems, Man, and Cybernetics 8.6 (1978): 460-473.

70. Suen, Ching Y., Marc Berthod, and Shunji Mori. "Automatic recognition of handprinted characters-the state of the art." Proceedings of the IEEE 68.4 (1980): 469-487.

71. Ogawa, Hideo, and Keiji Taniguchi. "Thinning and stroke segmentation for handwritten Chinese character recognition." Pattern recognition 15.4 (1982): 299-308.

72. Smith, Raymond W. "Computer processing of line images: A survey." Pattern recognition 20.1 (1987): 7-15.

73. Lam, Louisa, and Ching Y. Suen. "Structural classification and relaxation matching of totally unconstrained handwritten zip-code numbers." Pattern recognition 21.1 (1988): 19-31.

74. Abdulla, Waleed Habib, Aladdin OM Saleh, and A. H. Morad. "A preprocessing algorithm for hand-written character recognition." Pattern Recognition Letters 7.1 (1988): 13-18.

75. Lam, Louisa, Seong-Whan Lee, and Ching Y. Suen. "Thinning methodologies-a comprehensive survey." IEEE Transactions on pattern analysis and machine intelligence 14.9 (1992): 869-885.

76. Rahier, Michel C., and Paul G. A. Jespers. "Dedicated LSI for a microprocessor-controlled hand-carried OCR System." IEEE Journal of Solid-State Circuits 15.1 (1980): 15-24.

77. Rieck, G. W., and K. Kratzheller. "Zytologie des desquamierenden Vaginalepithels der Hündin undihre Anwendung für die Zyklusdiagnostik." Transboundary and Emerging Diseases 2.1 (1955): 82-101.

78. Kirsch, Russell A., et al. "Experiments in processing pictorial information with a digital computer." Papers and discussions presented at the December 9-13, 1957, eastern joint computer conference: Computers with deadlines to meet. ACM, 1957.

79. O'Gorman, Lawrence, and Rangachar Kasturi. Document image analysis. Vol. 39. Los Alamitos: IEEE Computer Society Press, 1995.

80. Ahmed, Maher, and Rabab Ward. "A rotation invariant rule-based thinning algorithm for character recognition." IEEE Transactions on Pattern Analysis and Machine Intelligence 24.12 (2002): 1672-1678.

81. Ahmed, Maher, and Rabab Ward. "A rotation invariant rule-based thinning algorithm for character recognition." IEEE Transactions on Pattern Analysis and Machine Intelligence 24.12 (2002): 1672-1678.

82. Favata, John T., and Geetha Srikantan. "A multiple feature/resolution approach to the handprinted digit and character recognition." International journal of imaging systems and technology 7.4 (1996): 304-311.

83. Khorsheed, Mohammad S. "Off-line Arabic character recognition-a review." Pattern analysis \& applications 5.1 (2002): 31-45.

84. Shaw, Bikash, Ujjwal Bhattacharya, and Swapan K. Parui. "Combination of features for efficient recognition of offline handwritten Devanagari words." Frontiers in Handwriting Recognition (ICFHR), 2014 14th International Conference on. IEEE, 2014.

85. O'Gorman, Lawrence, and Rangachar Kasturi. Document image analysis. Vol. 39. Los Alamitos: IEEE Computer Society Press, 1995.

86. Liou, Cheng-Yuan, and Hsin-Chang Yang. "Handprinted character recognition based on spatial topology distance measurement." IEEE Transactions on Pattern Analysis and Machine Intelligence 18.9 (1996): 941 -945.

87. Sharma, Nabin, et al. "Recognition of off-line handwritten devnagari characters using quadratic classifier." Computer Vision, Graphics and Image Processing. Springer Berlin Heidelberg, 2006. 805-816.

88. Pal, Umapada, et al. "Handwritten numeral recognition of six popular Indian scripts." Document Analysis and Recognition, 2007. ICDAR 2007. Ninth International Conference on. Vol. 2. IEEE, 2007.

89. Bhattacharya, Ujjwal, and Bidyut Baran Chaudhuri. "Handwritten numeral databases of Indian scripts and multistage recognition of mixed numerals." IEEE transactions on pattern analysis and machine intelligence 31.3 (2009): 444-457.

90. Patra, Prashant Kumar, et al. "Probabilistic neural network for pattern classification." Neural Networks, 2002. IJCNN'02. Proceedings of the 2002 International Joint Conference on. Vol. 2. IEEE, 2002.

91. Patil, S. Basavaraj, and N. V. Subbareddy. "Neural network based system for script identification in Indian documents." Sadhana 27.1 (2002): 83-97.

92. Liou, Cheng-Yuan, and Hsin-Chang Yang. "Handprinted character recognition based on spatial topology distance measurement." IEEE Transactions on Pattern Analysis and Machine Intelligence 18.9 (1996): 941-945. 
93. Nawaz, Syed Nazim, et al. "An approach to offline Arabic character recognition using neural networks." Electronics, Circuits and Systems, 2003. ICECS 2003. Proceedings of the 2003 10th IEEE International Conference on. Vol. 3. IEEE, 2003.

94. Liou, Cheng-Yuan, and Hsin-Chang Yang. "Handprinted character recognition based on spatial topology distance measurement." IEEE Transactions on Pattern Analysis and Machine Intelligence 18.9 (1996): 941-945.

95. Arora, Sandhya, et al. "Combining multiple feature extraction techniques for handwritten devnagari character recognition." Industrial and Information Systems, 2008. ICIIS 2008. IEEE Region 10 and the Third international Conference on. IEEE, 2008.

96. Rajashekararadhya, S. V., and P. Vanaja Ranjan. "Efficient zone based feature extration algorithm for handwritten numeral recognition of four popular south Indian scripts." Journal of Theoretical \& Applied Information Technology 4.12 (2008).

97. Sharma, Divya. Recognition of Handwritten Devanagari Script Using Soft Computing. Diss. THAPAR UNIVERSITY PATIALA, 2009.

98. Jain, Anil K., M. Narasimha Murty, and Patrick J. Flynn. "Data clustering: a review." ACM computing surveys (CSUR) 31.3 (1999): 264-323.

99. Battiato, Sebastiano, et al. "An efficient algorithm for the approximate median selection problem." Algorithms and complexity (2000): 226-238.

100.Uchida, Seiichi, and Hiroaki Sakoe. "A survey of elastic matching techniques for handwritten character recognition." IEICE transactions on information and systems 88.8 (2005): 1781-1790.

101. Mohamad, Muhammad'Arif, et al. "A review on feature extraction and feature selection for handwritten character recognition." International Journal of Advanced Computer Science \& Applications 1.6 (2015): 204212.

102. Sangwan, Sandeep Singh, Er Sukhvinder Kaur, and Dinesh Arora. "An Efficient Fully Parallel Skeletonization Algorithm for Device Independent BMP Images."

103.Zou, Ju Jia, and Hong Yan. "Skeletonization of ribbon-like shapes based on regularity and singularity analyses." IEEE Transactions on Systems, Man, and Cybernetics, Part B (Cybernetics) 31.3 (2001): 401-407.

104.H. Chatbri and K. Kameyama, "Using Scale Space Filtering to Make Thinning Algorithms Robust Against Noise in Sketch Images", International Conference on Pattern Recognition letters Elsevier, vol. 42, (2014), pp. $1-10$.

105.Guo Z. and Hall R.W., "Parallel Thinning with Two-Sub Iteration Algorithms", Communications of the Association of Computer Machinery (ACM) Image Processing and Computer Vision, vol. 32, no. 3, (1989), pp.359-373.

106. T. Y. Zhang and C. Y. Suen, “A Fast-Parallel Algorithm for Thinning Digital Patterns”, Communications of the Association for Computer Machinery (ACM), vol. 27, no. 3, (1984), pp. 236-239.

107.R. W. Zhou, C. Quek and G. S. Ng, "A Novel Single-Pass Thinning Algorithm and an Effective Set of Performance Criteria”, International Journal of Pattern Recognition Letters Elsevier, vol. 16, Issue 12, (1995), pp. 1267-1275.

108.M. Ahmed and R. Ward, “A Rotation Invariant Rule-Based Thinning Algorithm for Character Recognition", IEEE Journal on Pattern Analysis and Machine Intelligence, vol. 24, no. 12, (2002), pp. 1672-1678.

109.P. I. Rockett, “An Improved Rotation-Invariant Thinning Algorithm”, IEEE Journal on Pattern Analysis and Machine Intelligence, vol. 27, no. 10, (2005), pp. 1671-1674.

110.P. Tarabek, "Performance Measurements of Thinning Algorithms", Journal of Information, Control and Management Systems, vol. 6, no. 2, (2008), pp. 125-132.

111.G.V. Padole and S. B. Pokle, "New Iterative Algorithms for Thinning Binary Images", IEEE Third International Conference on Emerging Trends in Engineering and Technology, vol. 7, (2010), pp. 166-171.

112.A. Jagna and V. Kamakshiprasad, "New parallel binary image thinning algorithm", ARPN Journal of Engineering and Applied Sciences, vol. 5, no. 4, (2010), pp. 64-67

113.J. Kwon, "Improved Parallel Thinning Algorithm to Obtain Unit -Width Skeleton", The International Journal of Multimedia \& Its Applications (IJMA), vol. 5, no. 2, (2013), pp. 1-14.

114. Sharma N, Pal U, Kimura F and Pal S: Recognition of offline handwritten Devanagari characters using a quadratic classifier, In Computer Vision, Graphics and Image Processing Conference, Springer Berlin Heidelberg, 2006, pp. 805-816.

115.Deshpande P S, Malik L and Arora S: Fine classification \& recognition of handwritten Devnagari characters with regular expressions \& minimum edit distance method, Journal of Computers, vol. 3, no. 5, 2008, pp. 11-17. 
116. Xue, H. A. N. H. O. N. G., and Venu Govindaraju. "Character recognition by matching sequences of pseudostroke positions and directions." Proceedings of the Seventh International Workshop on Frontiers in Handwriting Recognition. 2000.

117. Arora Sandhya, Bhattacharjee Debotosh, Nasipuri Mita, and Nagpur L. M.: A Two-Stage Classification Approach for Handwritten Devanagari Characters, IEEE International Conference on Computational Intelligence and Multimedia Applications, vol. 2, 2007, pp. 399-403.

118. Bajaj, Reena, Lipika Dey, and Santanu Chaudhury. "Devanagari numeral recognition by combining decision of multiple connectionist classifiers." Sadhana 27.1 (2002): 59-72.

119. Hanmandlu, Madasu, OV Ramana Murthy, and Vamsi Krishna Madasu. "Fuzzy Model based recognition of handwritten Hindi characters." Digital Image Computing Techniques and Applications, 9th Biennial Conference of the Australian Pattern Recognition Society on. IEEE, 2007.

120. Arora S, Bhattacharjee D, Nasipuri M, Basu D K and Kundu M: Recognition of non-compound handwritten Devnagari characters using a combination of MLP and minimum edit distance, International Journal Computer Science Security, vol. 4, no. 1, 2010, pp. 107-120.

121.S. Kumar: Performance comparison of features on Devnagari hand printed dataset, International Journal on Recent Trends, vol. 1, no. 2, 2009, pp. 33-37.

122.Pal, Umapada, Tetsushi Wakabayashi, and Fumitaka Kimura. "Comparative study of Devnagari handwritten character recognition using different feature and classifiers." Document Analysis and Recognition, 2009. ICDAR'09. 10th International Conference on. IEEE, 2009.

123.Pal, Umapada, Tetsushi Wakabayashi, and Fumitaka Kimura. "Handwritten Bangla compound character recognition using gradient feature." Information Technology, (ICIT 2007). 10th International Conference on. IEEE, 2007.

124.Jangid, Mahesh. "Devanagari isolated character recognition by using statistical features." International Journal of Computer Science and Engg 3.2 (2011): 2400-2407.

125.S. Shelke, "A Multistage Handwritten Marathi Compound Character Recognition Scheme using Neural Networks and Wavelet Features," vol. 4, no. 1, pp. 81-94, 2011.

126. Sharma N, Pal U, Kimura F, and Pal S. Recognition of off-line handwritten Devnagari characters using quadratic classifier, International conference on Computer Vision, Graphics and Image Processing, Springer Berlin Heidelberg, 2006, pp. 805-816.

127. Pal U, Roy R K, Roy K and Kimura F. Indian multi-script full pin-code string recognition for postal automation, IEEE International Conference on Document Analysis and Recognition, 2009, pp. 456-460.

128..Bhattacharya U and Chaudhuri B B. Handwritten numeral databases of Indian scripts and multistage recognition of mixed numerals, IEEE Transactions on Pattern Analysis and Machine Intelligence, vol. 31, no. 3, 2009, pp. 444-457. 